\title{
Characterization of Dielectric Waveguides Through Photoemission Electron Microscopy (PEEM) in the Infrared Regime
}

\author{
T. A. Stenmark ${ }^{1}$, Robert C. Word ${ }^{1}$ and R. Könenkamp ${ }^{1}$ \\ ${ }^{1}$ Department of Physics, Portland State University, Portland, OR 97201, USA
}

Photoemission Electron Microscopy (PEEM) provides a powerful tool for imaging electric fields and plasmon excitation. PEEM relies on the photoelectric effect with the photoemission yield directly dependent on the intensity of the electric field at the surface of the object being imaged. These properties provide valuable information about diffraction and interference as well as surface plasmon propagation and photo emission thresholds. High power pulse lasers allow for multiphoton PEEM where multiple photons are required in order to emit a single electron. These techniques produce images with resolution down to around $5 \mathrm{~nm}$ in the visible and infrared regime [1]. It is therefore possible to directly image the near-field region of the electromagnetic field [2]. We report here, for the first time, the direct observation polarization dependent phase shifts in the in-coupling of light in the $780 \mathrm{~nm}$ regime at the edge of a linear dielectric waveguide. Using finite element techniques we show that these phase shifts are correctly predicted. Furthermore the obtained photoemission micrographs allow a determination of optical properties on the nanometer scale, i.e. optical near-field distributions can be quantified and energy transfer and loss process can be analyzed. This opens the doors to sub-wavelength and dynamic imaging in metamaterial devices.

In our experimental setup the substrate is a $0.2 \mathrm{~mm}$ thick glass platelet covered by an indium-tin-oxide (ITO) layer approximately $290 \mathrm{~nm}$ thick. The greater refractive index of the ITO layer creates a twodimensional waveguide. An FEI Strata 237 focused ion beam was used to define a strip waveguide of 2 um width and milled 50nm into the ITO layer. In this strip waveguide only discrete set of modes can be excited. These modes create an interference pattern with the incident light. For the in-coupling of light into the guide a rectangular slit was milled at the edge of the strip waveguide in a direction perpendicular to the excitation light, as shown in Fig. (1) A general analysis of the waveguide performance has been carried out recently [3]. These results are extended here to include the nearinfrared region, where a 3-photon process is needed to generate a free photoelectron.

By taking the Fourier transform of the intensity distribution along the length of the waveguide and extracting the periodic information (fig 2) we are able to determine distinct single modes for both TE and TM polarizations for 780nm illumination and calculate the effective indexes of refraction for these modes. The resulting effective indexes are 1.596 for TE and 1.544 for TM which translates to a thickness of approximately 250nm according to numerical calculations [4] (fig 3).

Additional simulations are highly consistent with numerical calculations matching effective indexes to $0.2 \%$ error. Our new results demonstrate the possibility of obtaining images with nanometer resolution for optical phenomena across the entire visible spectrum. These possibilities show PEEM to be an efficient tool for analyzing and modeling photon [3] and plasmon [5] dynamics and interactions at solid surfaces and devices. 


\section{References}

[1] JPS Fitzgerald, RC Word, R Könenkamp, Phys. Rev. B 89 (2014), 195129.

[2] (6) Livio I. Chelaru and F.-J. Meyer zu Heringdorf, Appl. Phys. Lett. 89, 241908 (2006)

[3]JPS Fitzgerald, RC Word, R Könenkamp, Phys. Rev. B87 (2013), 205419.

[4]A. Yariv, Optical Electronics (Saunders College Publishing, Philadelphia, 1991)

[5] Niemma M. Buckanie et al., Ultramicroscopy 130, 49 (2013)
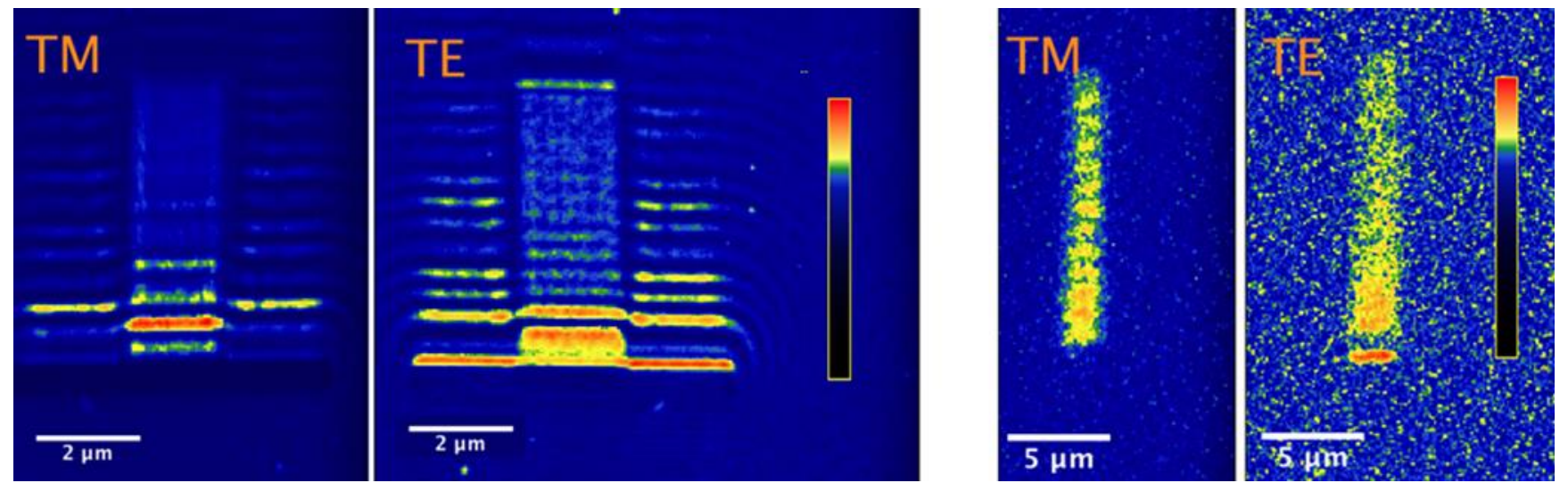

Figure 1: Colorized PEEM images. 410nm illumination on the left and 780nm on the right. Note the noisier image due to reduced signal at the lower energy wavelength.

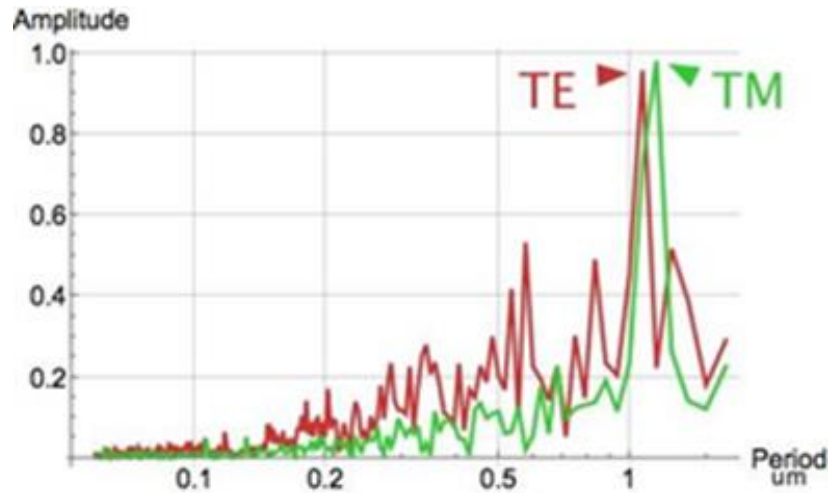

Figure 2: Periodic data of the interference pattern. TE peak at $1.07 \mathrm{um}$, TM $1.15 \mathrm{um}$

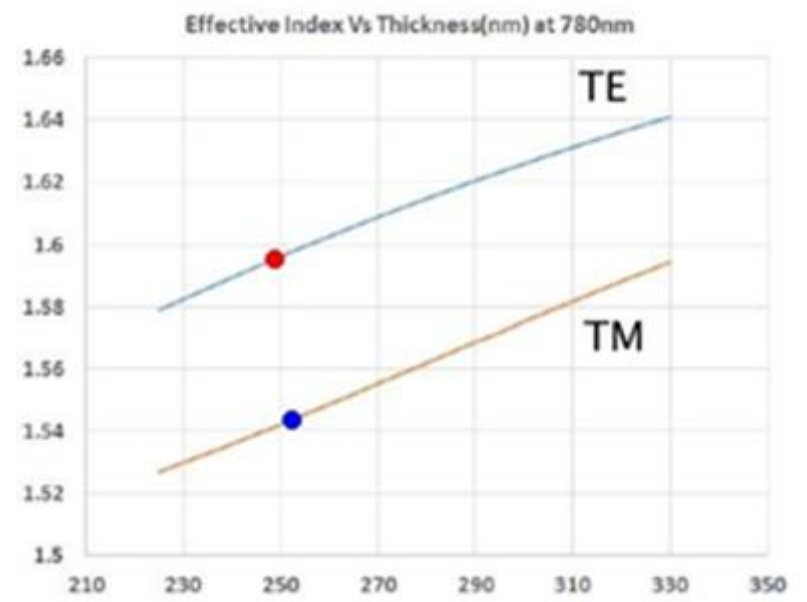

Figure 3: Numerical calculation of Effective index vs waveguide Thickness For ITO on a glass substrate at $780 \mathrm{~nm}$. Dots are where the experimental values for Neff fall on the graph. 